\title{
A-ESDT: Energy Saving and Stable Efficiency Mechanism for Cellular Interface in Smart Phones
}

\author{
Li Mingli ${ }^{1, a}$, Li Taoshen ${ }^{1, b}$ and Ge Zhihui ${ }^{1, c}$ \\ ${ }^{1}$ School of Computer, Electronics and Information in Guangxi University, Nanning, Guangxi, China \\ aImlflysky44@163.com, btshli@gxu.edu.cn, 'cgezhihui@gxu.edu.cn
}

Keywords: the third generation(3G);radio resource control(RRC); tail energy; cellular interface

\begin{abstract}
The frequency and micro scale characteristics of data transmission in smart phones increase the frequency of occurrence and proportion of tail energy, which decreases the utilization rate of transmission energy. In this paper, an aggregate-based energy saving data transmission mechanism (A-ESDT) is proposed, it adopts the opportunity cost of transmission to optimize the instability. This mechanism takes data transmission types and cellular port recent RRC state together to decide whether transmits the data now or does it later. Experimental result shows that the A-ESDT mechanism enhance the energy efficiency compared to the normal mechanism.
\end{abstract}

\section{Introduction}

With the popularity of the Mobile Internet and maturity of the smart phones, applications in smart phone basically need to exchange data with Internet. The most important characteristic of transfer energy in communicate network is that it mainly consists of tail energy. In order to avoid "Signaling Storm" caused by cellular interface in smart phones frequently switch its RRC state, the operators set inactive timer in mobile communicate network. When each data transmission completes, cellular interface still stays in high power state for a period of time waiting for the inactive timer count down, so the cellular interface can switch to a lower power state for energy saving. Any new data transmission occurring in the period of time makes the inactive timer recount again. The energy consumed in that time calls tail energy and it takes above $60 \%$ of the total transfer energy ${ }^{[1]}$. Since mostly traffics generate their own tail energy after transmission, it's helpful to reduce transfer energy consumption by aggregating small packets into a big one, so that they can share one tail energy ${ }^{[2]}$.

Aggregation in transmission is usually based the data classification and cellular interface RRC state. There are two different types of data classified by its tolerant time. The one needs transfer immediately calls instant type with no tolerant time; another one can transfer in the future calls delay-tolerant type with tolerant time spans from minutes to hours. The normal aggregation working mechanism is as follows:

1) If the data is instant type, transfers it at once without consideration of cellular interface RRC state.

2) If the data is delay-tolerant type, does not transfer until it reaches its deadline or the cellular interface is in high power state again.

The simplicity of transmission decision may result in instability of energy saving efficiency. For example, a new delay-tolerant type of data arrives at the end of tail time. Because the cellular interface is still in high power state, it has the right to transfer immediately. This transmission behavior resets the inactive timer, so the tail energy has been consumed before the transmission is wasted. In the case that data transmissions have intense interval, the simple aggregation results in instable energy saving efficiency.

In order to improve this situation, this paper proposes a data transmission mechanism based on the idea of aggregation (Aggregate-based Energy Saving Data Transmission, A-ESDT). This mechanism balances the opportunity cost to make the decision that transfers the new arrival delay-tolerant type of data or not.

The rest of this paper is organized as follows. Section 2 describes related research works.Section 3 elaborates the design of A-ESDT mechanism, including the computation of opportunity cost and 
waiting queue. Section 4 presents the experiment environment and the experimental results between the A-ESDT mechanism. Section 5 gives the conclusion of this paper.

\section{Related Works}

Background Knowledge. The instability of energy saving efficiency comes from the inappropriate judgment on delay-tolerant type of data transmission. This paper takes 3G communication network as example. There are three major RRC states in 3G for cellular interface. Figure 1 shows RRC state machine in 3G communicate network.

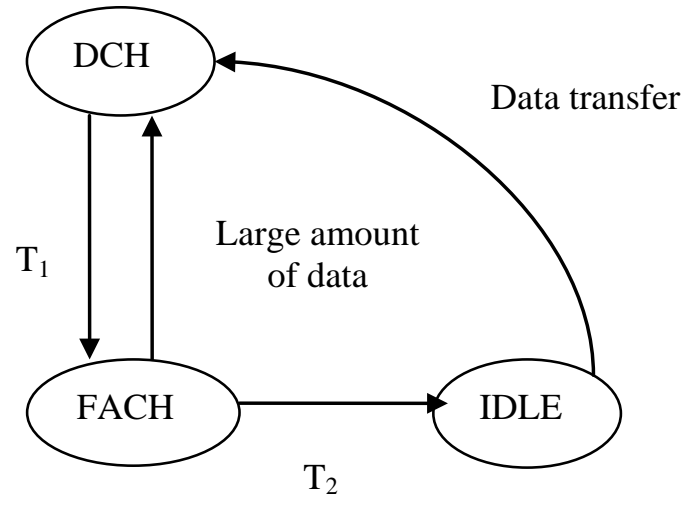

Figure 1: RRC state machine in 3G communicate network

There are two inactive timers in $3 \mathrm{G}$ communicate network, $\mathrm{T}_{1}$ is in charge of switching from DCH state to FACH state, $\mathrm{T}_{2}$ from $\mathrm{FACH}$ state to IDLE state. A complete tail energy is energy consumption in time $\mathrm{T}_{1}$ and $\mathrm{T}_{2}$, which means that cellular state switches from DCH to IDLE.

Related Works. In order to reduce tail energy generating in communicate network, some researchers use to aggregate the traffic of smart phones to transfer together. This strategy lets several traffic share one tail energy to achieve energy saving.Niranjan et al.[1] have designed a TailEnder protocol that can save energy for delay-tolerance delay tolerance-program. This protocol is especially suitable for small and medium sized e-mail, and its energy efficiency is achieved by $50 \%$. However, it does not take into account the Web browser or instant messaging which data transformation are more frequently. Wang et al.[2] improved the Web browsing transport mechanism of smart phone, and have proposed a new mechanism to aggregate the multiple data transfers as a transfer. But this method does not take into account the problem of user-control option. Aggarwal et al.[3] have combined cloud computing technologies and mobile network energy-saving strategies, and designed a prototype system called Stratus. The Stratus uses a proxy mechanism based on cloud computing servers, and migrates phone direct communication with remote server to the cloud server. This method is simple, and its energy efficiency is significant. But it only applicable to down-link aggregation transmission and require additional proxy server overhead. Ref. [4] and [5] analyzed the existing transport protocol, and have pointed out that LTE's energy efficiency is lower in terms of transferring trace data, and have made different improvements to the transport protocol from from the aggregate transformation respectively.

In general, for delay-tolerance programs, the energy-saving efficiency of aggregate transformation methods is higher. However, for common instant message tool used to smart phones and the processes needed to maintain the long link state, simple aggregation may lead to unstable energy saving efficiency.

\section{A-ESDT Mechanism}

Design Idea. The design idea of A-ESDT mechanism design idea is as follows: in order to improve energy-saving efficiency of the smart phone, multiply delay-tolerance data transforming requests are bundled together with aggregate transport mechanism, and then send. This mechanism takes data transmission types and cellular port recent RRC state together to decide whether transmits the data now or does it later. 
In the algorithm design, the following aspects are considered:

Waiting Queue. A-ESDT constructs a waiting queue for the delay-tolerant type of data. When the data do not meet the transfer condition, they join into the waiting queue. Data in waiting queue are sorted by their deadline from the earliest to the latest.

Opportunity Cost. Opportunity cost means that the most value you should pay for doing the other thing. In the case of transmission, opportunity cost is loss of the tail energy already consumed; in the case of joining into waiting queue, opportunity cost is the generation of a complete tail energy when it fails to aggregate with other data in its deadline.

Data Classification. This paper divides data transmission into three types, that is the instant type, the heartbeat type and the delay-tolerant type. Instant type and delay-tolerant type's definition is the same as above. The heartbeat type of data also has tolerant time, but it's shorter. And heartbeat type has periodicity as its particular characteristic. Heartbeat type of data frequently occurs in the scene that application keep TCP link in long connection with the remote server.

Aggregation Probability. The computation of aggregation probability is the core of A-ESDT. It needs to compute the probability that all of the three type transfer in the deadline of new arrival heartbeat/delay-tolerant data. The transfer probability is decide by the sampling interval of the instant type data, the periodicity of the heartbeat type of data and the earliest deadline in the waiting queue:

a) Taking into account that when people use application to chat or watch video, the interval time of data transmission should have certain regularity. We use the thought of BP neural network algorithm ${ }^{[6]}$ which is popular for time sequence prediction to forecast the time of next instant type of data transmission request appears.

b) Because the heartbeat type of data has the strong quality of periodicity, we add up the past heartbeat data and find how many applications generate them.

c) If the earliest deadline in waiting queue is earlier than the new arrival data transmission deadline, the transfer probability is 1 , or else 0 .

The actual transfer probability is the maximum value of the probabilities than calculated by three methods above.

The A-ESDT Algorithm. The A-ESDT algorithm is as follows:

1: $\mathrm{D}_{\text {type }}$ : the data type specified above

2: $\mathrm{CI}_{\text {state }}$ : the cellular interface state

3: while ture do

4: if $\mathrm{D}_{\text {type }}$ is instant then

5: $\quad$ transfer this data request;

6: $\quad$ transfer the waiting queue;

7: else

8: $\quad$ if $\mathrm{CI}_{\text {state }}$ is $\mathrm{DCH}$ or $\mathrm{FACH}$ then

9: $\quad$ transfer this data request;

10: $\quad$ transfer the waiting queue;

11: else

12: $\quad$ join this data request into waiting queue;

13: $\quad$ End if

14: end if

15: end while

The A-ESDT does not only make transmission judgment by cellular interface RRC state and the type of data, it balances the opportunity cost which is decided by transfer probability to make a decision. In this way, A-ESDT can avoid the phenomenon that new arrival data transfer when the inactive timer almost count down, the new transmission makes the inactive timer recount again and almost double the tail time. 


\section{Experimentation and Algorithm Analysis}

Experimental environment. In this section, we evaluate the performance of normal aggregation and A-ESDT through the trace-driven simulation. The traces generate by a exponential distribution generator according to Maier $\mathrm{G}^{[7]}$. There is $30 \%$ of traffic size is less than $1 \mathrm{~Kb}, 70 \%$ less than $3 \mathrm{~Kb}$, and the medium size is $24 \mathrm{~Kb}$. The average of downlink to uplink ratio is 6 to1. And cellular interface RRC state's power and switch time come from Huang $\mathrm{J}^{[8]}$. The parameters are measured at HTC G1 in 3G communicate network. Table 1 shows the parameters of RRC state machine in 3G communicate network:

Table 1: RRC state machine parameters of HTC G1 in the 3G communicate network ${ }^{[8]}$

\begin{tabular}{|c|c|c|}
\hline & Power & Inactive timer or time \\
\hline DCH & 804 & 8088 \\
\hline FACH & 601 & 824 \\
\hline IDLE & 11 & - \\
\hline IDLE——DCH & 659 & 582 \\
\hline
\end{tabular}

The transmission speed is set to $500 \mathrm{Kpbs}$ in downlink and 200Kpbs in uplink in accordance with actual 3G communicate network rate in China.

Experimental Results and Performance Analysis. Table 2 shows the experimental results of each part of transmission energy between normal transfer mechanism and the A-ESDT mechanism In $3 G$ or LTE network respectively. In Table 2, the unit of energy consumption is the $\mathrm{mW} /$ hour(mWh).

Table 2: The experimental results of transmission energy between normal mechanism and A-ESDT mechanism in 3G and LTE network

\begin{tabular}{|c|c|c|c|c|}
\hline & \multicolumn{2}{|c|}{ 3G network } & \multicolumn{2}{c|}{ LTE network } \\
\cline { 2 - 5 } & Normal mechanism & A-ESDT mechanism & Normal mechanism & A-ESDT mechanism \\
\hline $\begin{array}{c}\text { Increasing energy } \\
\text { consumption }\end{array}$ & 0.22 & 0.146 & 0.177 & 0.118 \\
\hline $\begin{array}{c}\text { Transmission energy } \\
\text { consumption }\end{array}$ & 1.455 & 1.455 & 0.225 & 0.225 \\
\hline $\begin{array}{c}\text { Tail energy } \\
\text { consumption }\end{array}$ & 7.03 & 3.831 & 13.58 & 7.02 \\
\hline $\begin{array}{c}\text { Dormancy energy } \\
\text { consumption }\end{array}$ & 0.115 & 0.192 & 0.108 & 0.229 \\
\hline $\begin{array}{c}\text { Total energy } \\
\text { consumption }\end{array}$ & 8.956 & 5.687 & 14.09 & 7.735 \\
\hline
\end{tabular}

As can be seen from the experimental results, after 3G port has used A-ESDT mechanism to transport, increasing energy consumption was reduced by 33.5\%, tail energy consumption was reduced by $45.5 \%$, dormancy energy consumption has increased by $66.7 \%$, total energy consumption was reduced by $36.5 \%$. After LTE port has used A-ESDT mechanism to transport, increasing energy consumption was reduced by $33.5 \%$, tail energy consumption was reduced by $48.3 \%$, dormancy energy consumption has increased by $112.5 \%$, total energy consumption was reduced by $45.1 \%$. By contrast, after the aggregate packet transmission, LTE's energy-saving efficiency of total energy consumption is higher than 3G. This is because the tail energy consumption of LTE occupies a larger proportion of the total energy consumption. LTE port power is larger in tail energy stage, and its tail time is also longer, so the LTE has got more energy saving benefit after using aggregation transmission mechanism.But LTE's dormancy energy consumption is greater than 3G.

Figure 2 shows the total energy consumption in different number of data transmission requests.As it shows in Figure 4, the absolute value of the energy saving increase before 20 times, but slowly decrease after 50 times. 


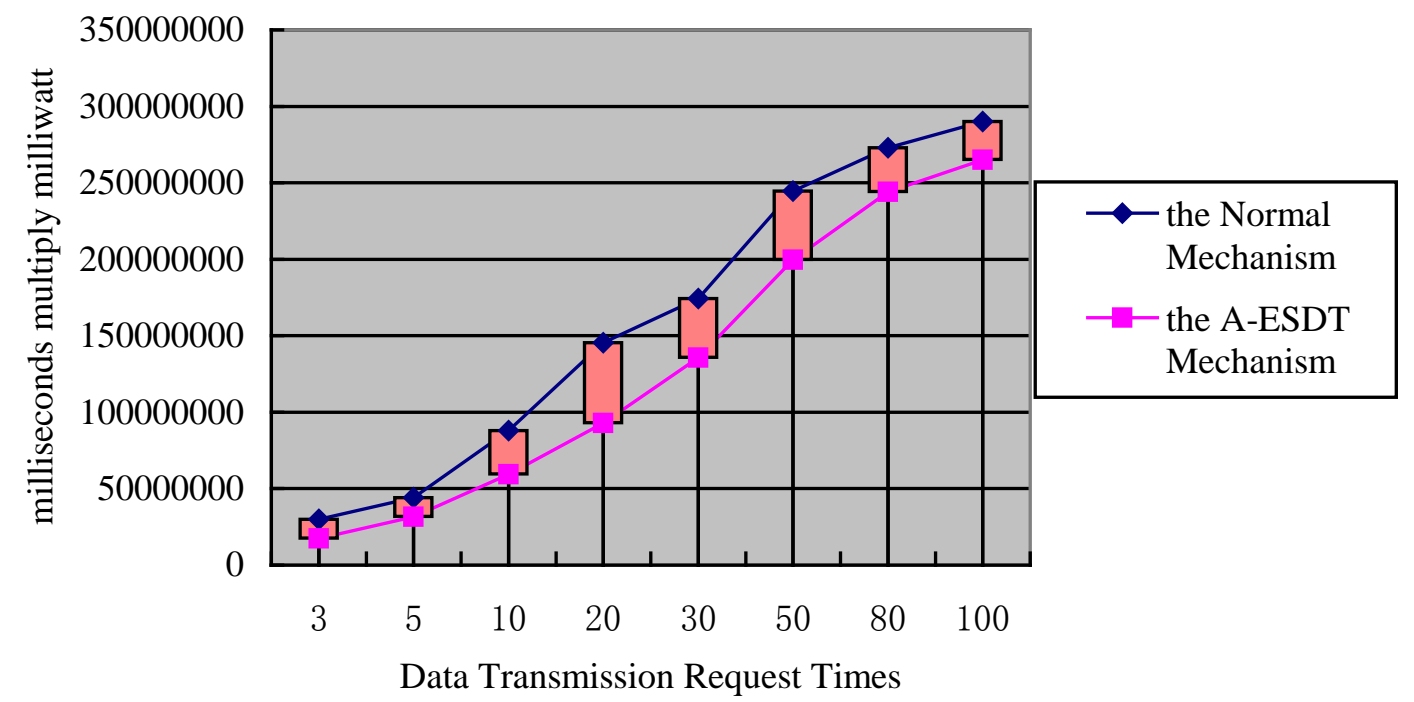

Figure 2: The comparison of total energy consumption between the normal and the A-ESDT mechanism in $3 \mathrm{G}$ among different data transmission request times

Figure 3 shows A-ESDT energy saving efficiency in different data transmission request times. Obviously, the its efficiency is high in before 30 times, but it is not stable after many times aggregation and decreases as the data transmission times increase. This problem needs to be further improved.

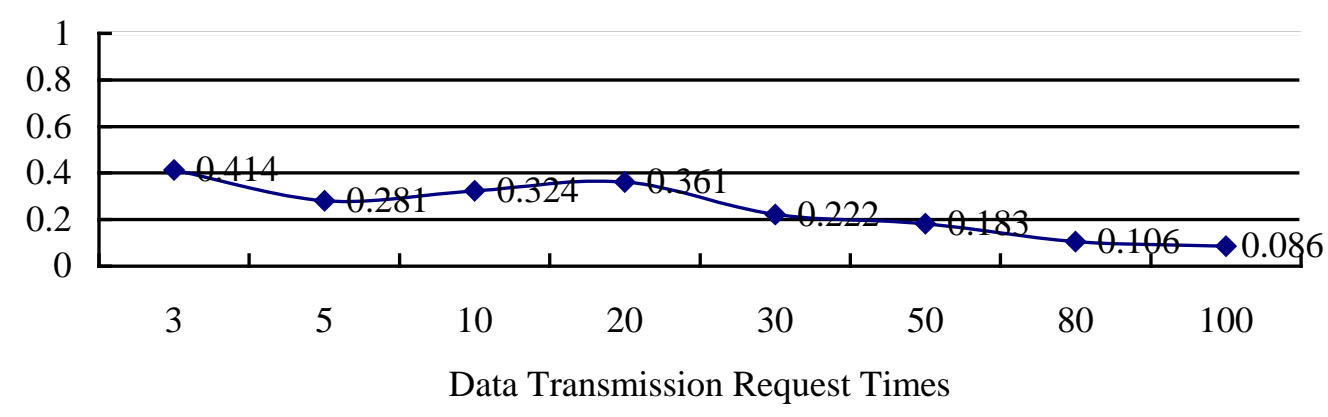

Figure 3: the energy saving efficiency of A-ESDT in Different Data Transmission Request Times

Through the analysis of the experimental results, we can draw a conclusion that A-ESDT's energy saving efficiency is excellent in the situation of small number of aggregations and large interval distribution of data requests. However its efficiency decreases along with large number of aggregations.

\section{Summary}

The tail energy generating after each data transfer complete takes a large proportion of total energy consumed in cellular interface. In this paper, we first introduce the A-ESDT mechanism, an idea to aggregate the data transmission together, let several transmissions share one tail energy. This mechanism takes cellular interface's RRC state and data transmission type into consideration to make the judgment of sending it immediately or joining it into the waiting queue. The A-ESDT mechanism achieves high energy saving efficiency when the data transmission requests' number is small, but the efficiency starts to decrease when the number grows bigger. Next, according to the A-ESDT mechanism problem, we will improve the A-ESDT algorithm by adding the concept of opportunity cost to balance the transmission decision of new arrival heartbeat/delay-tolerant data. 


\section{Acknowledgments}

This work is supported by the National Natural Science Foundation of China(Grant No 61363067).

\section{References}

[1] Balasubramanian N, Balasubramanian A, Venkataramani A. Energy consumption in mobile phones: a measurement study and implications for network applications[C]//Proceedings of the 9th ACM SIGCOMM conference on Internet measurement conference. ACM, 2009: 280-293.

[2] Wang L, Manner J. Energy-efficient mobile web in a bundle[J]. Computer Networks, 2013, 57(17): 3581-3600.

[3] Aggarwal B, Chitnis P, Dey A, et al. Stratus: energy-efficient mobile communication using cloud support[J]. ACM SIGCOMM Computer Communication Review, 2011, 41(4): 477-478.

[4] Wang K, Alonso-Zarate J, Dohler M. Energy-efficiency of LTE for small data machine-to-machine communications[C]//2013 IEEE International Conference on Communications (ICC),. IEEE Press, 2013: 4120-4124.

[5] Lauridsen M, Wang H, Mogensen P. LTE UE Energy Saving by Applying Carrier Aggregation in a HetNet Scenario[C]//2013 IEEE 77th Proceedings of Vehicular Technology Conference (VTC Spring), IEEE Press,2013: 1-5.

[6] Kasabov N K, Song Q. DENFIS: dynamic evolving neural-fuzzy inference system and its application for time-series prediction[J]. Fuzzy Systems, IEEE Transactions on, 2002, 10(2): 144-154.

[7] Maier G, Schneider F, Feldmann A. A first look at mobile hand-held device traffic[C]//Passive and Active Measurement. Springer Berlin Heidelberg, 2010: 161-170.

[8] Huang J, Qian F, Gerber A, et al. A close examination of performance and power characteristics of 4G LTE networks[C]//Proceedings of the 10th international conference on Mobile systems, applications, and services. ACM, 2012: 225-238. 